University of Wollongong

Research Online

Faculty of Business - Papers (Archive)

Faculty of Business and Law

$1-1-2015$

Sustainability and intertemporal equity: a multicriteria approach

Cinzia Colapinto

Ca'Foscari University of Venice

Danilo Liuzzi

University of Milan

Simone Marsiglio

University of Wollongong, simonem@uow.edu.au

Follow this and additional works at: https://ro.uow.edu.au/buspapers

Part of the Business Commons

Research Online is the open access institutional repository for the University of Wollongong. For further information contact the UOW Library: research-pubs@uow.edu.au 


\title{
Sustainability and intertemporal equity: a multicriteria approach
}

\begin{abstract}
In (macro)economics literature, the need to consider sustainability and intertemporal equity issues leads to propose different criteria (discounted utilitarianism, green golden rule, Chichilnisky criterion) in order to define social welfare. We compare and assess the outcomes associated to such alternative criteria in a simple macroeconomic model with natural resources and environmental concern (Chichilnisky et al. in Econ Lett 49:174-179, 1995), by relying on a multicriteria approach. We show that among these three criteria, the green golden rule (discounted utilitarianism) yields the highest (lowest) welfare level, while the Chichilnisky criterion leads to an intermediate welfare level which turns out to be increasing in the weight attached to the asymptotic utility. These results suggest that completely neglecting finite-time utilities and focusing only on the asymptotic utility is not only more sensible from a sustainability point of view but also from a social welfare maximization standpoint.
\end{abstract}

\section{Keywords}

intertemporal, equity, sustainability, multicriteria, approach

Disciplines

Business

Publication Details

Colapinto, C., Liuzzi, D. \& Marsiglio, S. (2017). Sustainability and intertemporal equity: a multicriteria approach. Annals of Operations Research, 251 (1-2), 271-284 


\title{
Sustainability and Intertemporal Equity: a Multicriteria Approach
}

\author{
Cinzia Colapinto* $\quad$ Danilo Liuzzi ${ }^{\dagger} \quad$ Simone Marsiglio ${ }^{\ddagger}$ \\ Forthcoming in Annals of Operations Research
}

\begin{abstract}
In (macro)economics literature, the need to consider sustainability and intertemporal equity issues leads to propose different criteria (discounted utilitarianism, green golden rule, Chichilnisky criterion) in order to define social welfare. We compare and assess the outcomes associated to such alternative criteria in a simple macroeconomic model with natural resources and environmental concern (Chichilnisky et al., 1995), by relying on a multicriteria approach. We show that among these three criteria, the green golden rule (discounted utilitarianism) yields the highest (lowest) welfare level, while the Chichilnisky criterion leads to an intermediate welfare level which turns out to be increasing in the weight attached to the asymptotic utility. These results suggest that completely neglecting finite-time utilities and focusing only on the asymptotic utility is not only more sensible from a sustainability point of view but also from a social welfare maximization standpoint.
\end{abstract}

Keywords: Sustainability, Intertemporal Equity, Social Welfare, Multicriteria

\section{Introduction}

Traditionally macroeconomics wishes to assess the impact of different events or policies on the wellbeing of the society as a whole. This is critical since it requires to understand how to define social welfare. The approach widely used in literature relies on the so-called discounted utilitarianism. According to such a criterion, social welfare coincides with the discounted sum of instantaneous per capita utilities. Such an approach is very convenient since it allows to state a canonical macroeconomic problem as an optimal control problem in which the objective function, coinciding with social welfare, is bounded and thus standard mathematical techniques can be borrowed and directly applied to find its solution. However the recent growing interest towards sustainability raises several questions about the effective ability of discounted utilitarianism to provide meaningful investigations of the underlying problems (see Heal, 2005). Even if the notion of sustainability is still controversial, the most widely spread definition has been provided by the World Commission on Environment and Development which labels sustainable development as that kind

\footnotetext{
${ }^{*}$ Ca' Foscari University of Venice, Department of Management, Venice, Italy. Email: cinzia.colapinto@unive.it

${ }^{\dagger}$ University of Milan, Department of Economics, Management and Quantitative Methods, Milan, Italy. Email: danilo.liuzzi@unimi.it

${ }^{+}$University of Wollongong, School of Accounting, Economics and Finance, Northfields Avenue, Wollongong 2522 NSW, Australia. Email: simonem@uow.edu.au

${ }^{1}$ Another critical aspect associated to the use of the utilitarian approach is related to the role of the population size and its eventual growth. Specifically, two different utilitarian approaches have been proposed in literature, the average (welfare coincides with individual or average utility) and total (welfare is the sum of individual utilities across the population) utilitarianism. See Palivos and Yip (1993) or more recently Marsiglio and La Torre (2012), Boucekkine and Fabbri (2013), and Marsiglio (2014) for a discussion of the implications of different utilitarian approaches. Since we abstract from population growth and normalize the population size, in our paper average and total utilitarianism coincide, thus we do not explicitly relate to this branch of the literature.
} 
of development satisfying "the needs of the present without compromising the ability of future generations to meet their own needs" (WCED, 1987). As it may be clear from such a definition, any sustainability discourse requires to carefully take into consideration two different aspects: respect of natural resources and intertemporal equity (Chichilnisky et al., 1995). Thus, the discounting utilitarianism, requiring to discount at a certain (generally constant) rate instantaneous utilities, is not compatible with intergenerational equity since it attaches less weight to future generations. Almost one century ago Ramsey (1928) recognized that "discounting of future utilities is ethically indefensible and arises purely from a weakness of the imagination" (Ramsey, 1928).

In order to fix the shortcoming related to discounted utilitarianism, several proposals have been advanced $2^{2}$ (Ramsey, 1928; von Weizcker, 1967). Probably the most interesting and discussed approaches are the green golden rule and the Chichilnisky criterion. The green golden rule defines social welfare as the asymptotic utility level thus allowing to determine the highest indefinitely maintainable utility level (Chichilnisky et al., 1995). The Chichilnisky criterion defines social welfare as a weighted average between the discounted utilitarianism and the green golden rule welfare (Chichilnisky, 1997). Despite its simplicity this latter notion generates several problems in order to identify optimal path $s^{3}$ (Figuieres and Tidball, 2012), thus understanding how to assess sustainability outcomes is still an open question. The goal of this paper is to shed some light on this issue by developing a multicriteria approach which will allow us to compare how the definition of social welfare might impact on the outcomes of alternative economic choices. We thus consider a simple model with natural resources and environmental concern, as in Chichilnisky et al. (1995), to assess the performance of these criteria to achieve sustainable outcomes. Differently from Chichilnisky et al. (1995), we abstract from capital accumulation and focus only on the interactions between consumption choices and the evolution of natural assets in order to emphasize the trade-off between the (short run) economic benefits and the (long run) environmental costs associated to human activities.

This paper proceeds as follows. Section 2 proposes our macroeconomic model, which is simply an optimal control problem with one state and one control variable, plus a scrap function depending on both the control and state variable (consumption level and stock of natural resources, respectively). Section 3 briefly reviews the rationale behind multicriteria analysis and focuses on the two approaches, namely the scalarization and the goal programming techniques, which we will then use to translate our dynamic macroeconomic problem into a multicriteria model. This is explicitly done in section 4, where we develop both the scalarized and goal programming versions of the multicriteria model we employ in order to assess the impact of alternative notions of social welfare on consumption, natural resources and welfare level; we also discuss the results of our analysis for a certain parametrization of the model, and compare the outcomes under the two alternative specifications of the multicriteria problem. We show that for a realistic set of parameter values a clear ranking (in terms of welfare achievements) of welfare criteria exists: the green golden rule yields the highest level of welfare, the Chichilnisky criterion an intermediate level, and the discounted utilitarianism the lowest welfare level. Section 5 as usual concludes and proposes directions for future research.

\section{The Model}

According to the traditional macroeconomic literature, we consider a Ramsey-type (1928) model of optimal growth where a benevolent social planner tries to maximize social welfare. Social welfare, $W$, is defined according to the Chichilnisky criterion (Chichilnisky, 1997), meaning that it is a weighted average between the discounted utilitarian and green golden rule approach. Time is continuous, the time horizon is infinite and the pure rate of time preference is denoted by $\rho>0$; the instantaneous utility function depends on the level

\footnotetext{
${ }^{2}$ Several other criteria have been proposed in literature but to a large extent they turn out to be ad hoc proposal or do not allow a direct comparison with discounted utilitarianism (Pezzey, 1997; Arrow et at., 2004; Marsiglio, 2011).

${ }^{3}$ Another interesting related work is Le Kama's (2001), showing that by choosing the green golden rule utility level as Ramsey's bliss point for the non-discounted problem the optimal utilitarian path converges to the green golden rule outcome.
} 
of consumption, $c_{t}$, and the stock of natural resources, $e_{t}$, and it is assumed to take the following isoelastic form: $u\left(c_{t}, e_{t}\right)=\frac{\left(c_{t} e_{t}^{\beta}\right)^{1-\sigma}-1}{1-\sigma}$, where $\sigma>0$ is the inverse of the intertemporal elasticity of substitution and $\beta \geq 0$ represents the weight of the environment in the planner's preferences (the green preferences parameter). Population is constant and its size is normalized to 1, thus aggregate and per capita variables coincide. Natural resources accumulate according to their renewal capacity, assumed to be logistic, but are depleted by consumption activities; the rate of natural regeneration is $r$ and $e^{c}$ denotes the carrying capacity of the environment (Chichilnisky et al., 1995). Given the initial level of natural resources, $e_{0}$, the planner's problem consists of choosing the consumption level in order to maximize social welfare by taking into account the dynamic evolution of natural assets:

$$
\begin{array}{rl}
\max _{c_{t}} & W=\theta \int_{0}^{\infty} \frac{c_{t}^{1-\sigma} e_{t}^{\beta(1-\sigma)}-1}{1-\sigma} e^{-\rho t} d t+(1-\theta) \lim _{t \rightarrow \infty} \frac{c_{t}^{1-\sigma} e_{t}^{\beta(1-\sigma)}-1}{1-\sigma} \\
\text { s.t. } & \dot{e}_{t}=r e_{t}\left(1-\frac{e_{t}}{e^{c}}\right)-c_{t}
\end{array}
$$

Note that the objective function (1) corresponds to the Chichilnisky criterion, in which social welfare is defined as the weighted average between the discounted utilitarian welfare $\left(\int_{0}^{\infty} u\left(c_{t}, e_{t}\right) e^{-\rho t} d t\right)$ and the green golden rule welfare $\left(\lim _{t \rightarrow \infty} u\left(c_{t}, e_{t}\right)\right)$. The parameter $\theta \in[0,1]$ represents the weight assigned to the discounted integral of utilities. Specifically, when $\theta=1(\theta=0)$ social welfare is defined according to the discounted utilitarian (green golden rule) criterion, while for any $\theta \in(0,1)$ social welfare takes into account both the discounted utilitarian and green golden rule approach. Since analytical solutions cannot be found (unless in the extreme cases in which $\theta=0$ or $\theta=1$ ), in order to understand the role of the parameter $\theta$ in determining the evolution path of consumption and natural resources, and thus the social welfare level, we need to rely on a multicriteria approach.

As we will see later, the problem above turns out to be a particular case of a more general problem that can be tackled with the help of the multiple criteria decision analysis, in which two alternative (the discounted utilitarian and green golden rule) criteria are simultaneously pursued. Specifically, the maximization problem in (1) and (2) can be recast as the following multicriteria problem:

$$
\max \quad W=\left[W_{D U}, W_{G G R}\right],
$$

where $W_{D U}$ and $W_{G G R}$ represent the discounted utilitarian $(D U)$ and green golden rule $(G G R)$ welfare criteria respectively, defined as follows:

$$
\begin{aligned}
W_{D U} & \equiv \int_{0}^{\infty} \frac{c_{t}^{1-\sigma} e_{t}^{\beta(1-\sigma)}-1}{1-\sigma} e^{-\rho t} d t \\
W_{G G R} & \equiv \lim _{t \rightarrow \infty} \frac{c_{t}^{1-\sigma} e_{t}^{\beta(1-\sigma)}-1}{1-\sigma},
\end{aligned}
$$

and the maximization problem is subject to the dynamical constraint in (2). This ability to transform our macroeconomic model into a simple multicriteria problem is very convenient since it allows us to borrow from the operational research literature in order to understand the impact of alternative notions of social welfare (i.e., different values of $\theta$ ) on consumption, natural resources and welfare levels.

\section{Multicriteria Decision Analysis}

Multiple criteria decision analysis (MCDA, also known as multiple criteria decision making, MCDM) explicitly considers multiple and conflicting criteria such as cost, price, quality, time, performance, and others, in complex decision-making contexts and provides an alternative to the classical cost-benefit analysis (a popular tool in the 1970s and the 1980s), extensively used in economics in order to compare alternative 
policies or projects. Decision aid tools aim at establishing formulations of propositions to be submitted to the judgment of a decision maker or a group of decision makers (the social planner in our macroeconomic framework). As in any decision, since we have to consider different points of view and perspectives (dealing with finance, human resources, security, quality, etc.), a multicriteria approach appears more suitable to describe all different components of a decision-making process. Indeed, structuring complex problems as multiple criteria models leads to take better decisions. Since the beginning of the modern MCDA discipline in the early 1960s, many approaches and methods have been developed, also supported by many advanced and computationally-efficient decision-making software. MCDA methods are applied wherever there are several alternatives, which must be ranked in accordance with their significance in respect with the aim of the research or where the best alternative among the available ones must be identified. In other words, the primary purpose of analysis is a search for a compromise solution (Guitoni and Martel, 1998). By focusing only on projects that involve compromises between environmental costs and economic benefits, applications of MCDA in different areas have been proposed, such as industrial development (Nijkamp and van Delft, 1977), environmental policy issues (Janssen, 2001; Gamper and Turcanu, 2007), sustainability assessment at macroeconomic level (Shmelev, 2011), and macroeconomic policy (André et al., 2009).

There exist several alternatives to deal with a MCDA context (see for example Sawaragi et al., 1985; and Steuer, 1986); in the sequel we will mainly focus on two of them, namely the scalarization technique and the goal programming (GP) approach. The former method, namely the scalarization technique, represents the easiest way to deal with a MCDA model. Scalarizing a MCDA problem consists of constructing a singleobjective optimization problem such that optimal solutions of the single-objective optimization problem are the Pareto optimal solutions of the MCDA problem (Hwang and Masud, 1979). Scalarizing functions play an essential role in this decision-making context: in literature several different scalarizing functions have been proposed based on different approaches and philosophies. Probably the simplest specification is the weighted scalarization, which simply attaches a specific weight to each of the criteria. Mathematically, if $f_{1}(x), f_{2}(x), \ldots, f_{p}(x)$ are $p$ criteria to be maximized, and $w_{i} \in[0,1]$ are weights such that $\sum_{i=1}^{p} w_{i}=1$, a weighted scalarized model takes the form:

$$
\max \sum_{i=1}^{p} w_{i} f_{i}(x)
$$

The optimal solution of the MCDA problem will be depending on the chosen weights, and vary with them. The latter approach, that is the GP technique, is one of the most well-known MCDA models in which the solution of the best compromise minimizes the absolute deviations between the achievement levels $f_{i}(x)$ and the aspiration levels $g_{i}, \forall i=1, \ldots, p$. In fact, given a certain aspiration level both positive, $\delta_{i}^{+}$, and negative, $\delta_{i}^{+}$, deviations are unwanted. It is an a priori method, which means that the information regarding the goals is first decided by the decision maker and then a solution is determined by minimizing the difference between the achievement levels and the corresponding goals. In other words the original objectives of the problem are transformed into constraints and the optimization of the deviations from the goals results indirectly to optimize the initial objectives. The first formulation of the GP model has been presented by Charnes et al. (1955), and Charnes and Cooper $(1959,1968)$. Among all different GP formulations the weighted GP model with satisfaction function is the most suitable for our purposes. In this framework the decision maker (social planner) compares the performances of every possible action through the satisfaction function $F_{i}\left(\delta_{i}\right)$ (for more details see Martel and Aouni, 1990). The decision maker wishes to maximize his/her satisfaction, thus the greatest deviations are associated with lowest degrees of satisfaction. The mathematical formulation of the weighted GP program with satisfaction function can be expressed as follows:

$$
\begin{array}{ll}
\max & Z=\sum_{i=1}^{p}\left[w_{i} F_{i}^{+}\left(\delta_{i}^{+}\right)+w_{i} F_{i}^{-}\left(\delta_{i}^{-}\right)\right] \\
\text {s.t. } & f_{i}(x)-\delta_{1}^{+}+\delta_{1}^{-}=g_{i}
\end{array}
$$




$$
\begin{array}{ll}
0 \leq \delta_{i}^{+} \leq \delta_{i \nu}^{+}, & \forall i=1, \ldots, p \\
0 \leq \delta_{i}^{-} \leq \delta_{i \nu}^{-}, & \forall i=1, \ldots, p
\end{array}
$$

In the above specification the parameters $\delta_{i \nu}^{+}$and $\delta_{i \nu}^{-}$represent veto thresholds, such that both positive and negative deviations, $\delta_{i}^{+}$and $\delta_{i}^{-}$, cannot exceed such threshold values, $\delta_{i \nu}^{+}$and $\delta_{i \nu}^{-}$respectively.

Both the scalarization and GP approaches can be used in order to define a multicriteria problem, allowing to deal with a model like ours in (1) and (2). They both are easy to implement and can be solved through some powerful mathematical programming software such as LINGO and CPLEX. However, they both have pros and cons. Specifically, some positive aspects of goal programming are its simplicity and ease of use, especially because of its applicability to real problems (Aouni and Kettani, 2001), and the ability to allow decision makers to have a better control on the decision making context, since they can explicitly set their preferences. Moreover, when the parameters are subject to noise or uncertainty the problem can be easily analyzed by relying on stochastic GP models (see Aouni et al. 2012a, 2012b, 2013; Aouni and La Torre, 2010). With respect to the weighted scalarization approach, goal programming is not always able to produce solutions that are Pareto optimal. In fact, the GP model is based on the so-called "satisfying philosophy", meaning that the optimization task is replaced by the satisfaction in reaching certain levels for each criterion.

We will use both these approaches to analyze our macroeconomic problem, showing that they will lead to qualitatively equivalent results. The multicriteria optimization problem, both it its scalarized and GP versions, is solved with LINGO 14; given the dynamic nature of the problem and thus its large number of variables, we do not report LINGO's solution in our paper but we summarize it by showing the (optimal) dynamic evolution of our control (consumption) and state (natural resources) variables.

\section{A Multicriteria Approach}

We now propose two different methods in order to deal with our problem in (1) and (2), based on the weighted scalarization technique and the weighted GP with satisfaction function, respectively. We first show how to use these two alternative methodologies in order to study our problem and then compare the solution obtained under these two formulations of the multicriteria problem. Note that, since in the dynamical problem (3) time is continuous, in order to perform our numerical simulations we need firstly to discretize the problem. Moreover, since the time horizon is infinite, we also approximate this by assuming that time is finite, with the final time $T$ being sufficiently remote in the future to be actually seen as a plausible simplification of an infinite horizon. Since the utility contribution to the integral decays exponentially with time, a final time as $T=100$ is enough to appropriately do so. It is however possible to show that the results are robust with respect to the choice of the final time $T$. Since our main goal in the paper consists of assessing the impact of the different notions of social welfare on the dynamics of consumption, natural resources and welfare level, in both the formulations of the problem, we emphasize the role of the parameter $\theta$ which allows us to distinguish the discounted utilitarian $(\theta=1)$, the green golden rule $(\theta=0)$ and the Chichilnisky $(0<\theta<1)$ criteria. Specifically, we analyze the behavior of the solution for different values of $\theta$ in the range $\theta \in[0,1]$.

A critical aspect of any simulation is related to the value of the parameters employed. When possible we rely on standard values traditionally used in macroeconomics literature, while when not possible we rely on broad estimates of the parameters. Specifically, the value of the parameters used in our simulations is summarized in Table 1 .

\begin{tabular}{|c|c|c|c|c|c|}
\hline$\sigma$ & $\rho$ & $\beta$ & $r$ & $e^{c}$ & $e_{0}$ \\
\hline 2 & 0.04 & 0.2 & 0.05 & 3 & 1 \\
\hline
\end{tabular}

Table 1: Parameter values employed in our simulations. 
The pure rate of time preference, $\rho$, and the inverse of the intertemporal elasticity of substitution, $\sigma$, are set equal to 0.04 and 2, respectively (Barro and Sala-i-Martin, 2004). The green preference parameter, $\beta$, as in Mohtadi (1996)is set equal to 0.2. Note however that different values of the green preference parameter would yield the same qualitative results, apart from the extreme case $\beta=0$ (no environmental concern), which is not interesting for our analysis. The parameter values corresponding to the stock of natural resources strongly depend on the specific resource we are analyzing. Since we focus on a wide concept of natural resources, thus encompassing a broad range of natural assets, we rely on broad estimates related to fishery and forestry which can describe quite well the notion of natural resource we are adopting in our model. The initial value of the stock of natural resources, $e_{0}$, is set equal to 1 for the sake of simplicity. The carrying capacity, $e^{c}$, is set equal to 3 ; such a value is chosen since being relatively close to the initial stock allows to better stress the nature of the economic and environmental trade off. Nevertheless the results are robust for different values of the carrying capacity parameter. We assume the rate of natural regeneration, $r$, to be equal to 0.05 (see Eliasson and Turnovsky, 2004).

\subsection{Scalarization Technique}

According to the scalarization technique, we scalarize the vector $W=\left[W_{1}, W_{2}\right]$ with the help of two weights, such that what we need to optimize is a traditional single-criterion problem:

$$
\max _{c_{t}} \quad W=w_{D U} W_{D U}+w_{G G R} W_{G G R}
$$

where $w_{D U}=\theta$ and $w_{G G R}=1-\theta$, represent the relative importance the decision maker (the social planner) assigns to each objective $W_{i}, i=D U, G G R$, while $W_{D U}$ and $W_{G G R}$ are defined in (4) and (5), respectively. Note that this specification is exactly equivalent to our problem in (1) and (2), in which the objective function corresponds to the Chichilnisky criterion. By proceeding with the discretization, the infinite integral in (4) is substituted with a finite sum, while the limit in (5) with the value taken by the utility function at the final time $T$; the dynamic constraint instead is substituted with a set of difference equations. Hence the problem to be solved numerically is the following:

$$
\begin{array}{ll}
\max _{c_{t}} & W=\theta \sum_{t=0}^{T} \frac{c_{t}^{1-\sigma} e_{t}^{\beta(1-\sigma)}-1}{1-\sigma} e^{-\rho t}+(1-\theta) \frac{c_{T}^{1-\sigma} e_{T}^{\beta(1-\sigma)}-1}{1-\sigma} \\
\text { s.t. } & e_{t+1}-e_{t}=r e_{t}\left(1-\frac{e_{t}}{e^{c}}\right)-c_{t} \quad \forall t=0, \ldots . T-1 \\
& 0=r e_{T}\left(1-\frac{e_{T}}{e^{c}}\right)-c_{T} \\
& e_{0} \text { given }
\end{array}
$$

Some comments on the equations (6) to (9) are needed. When the final time $T$ is reached the economy is frozen, meaning that a steady state has been achieved and thus the second addendum in the equation (6) can be interpreted as lasting forever, approximating the idea of the maximum indefinitely substainable level of utility. Therefore there is no dynamics after $T$, as described by the equation (8).

The results of our numerical simulations are shown in Figure 1 and Figure 2, Figure 1 shows the optimal path of consumption and natural resources for different values of $\theta$, ranging from 0 to 1 , corresponding to social welfare functions relying on different approaches, varying from the green golden rule $(\theta=0)$ to the discounted utilitarianism $(\theta=1)$. The intermediate values of $\theta$ correspond to the Chichilnisky criterion, in which higher values of $\theta$ represent more importance attached to discounted utilitarianism. As the figure clearly shows, the closer $\theta$ to 0 , the more parsimonious is the consumption path and the more abundant the long run level of natural resources. Intuitively, the more emphasis we place on the steady state utility, the more we favor environmental preservation rather than economic activities; we thus consume less and preserve more natural resources for the (remote) future. Note that in the figure, the green golden rule case 
$(\theta=0)$ is reported with a dashed line in order to remind us that this represents only a steady state level, thus there is no dynamics from 0 to $T$.
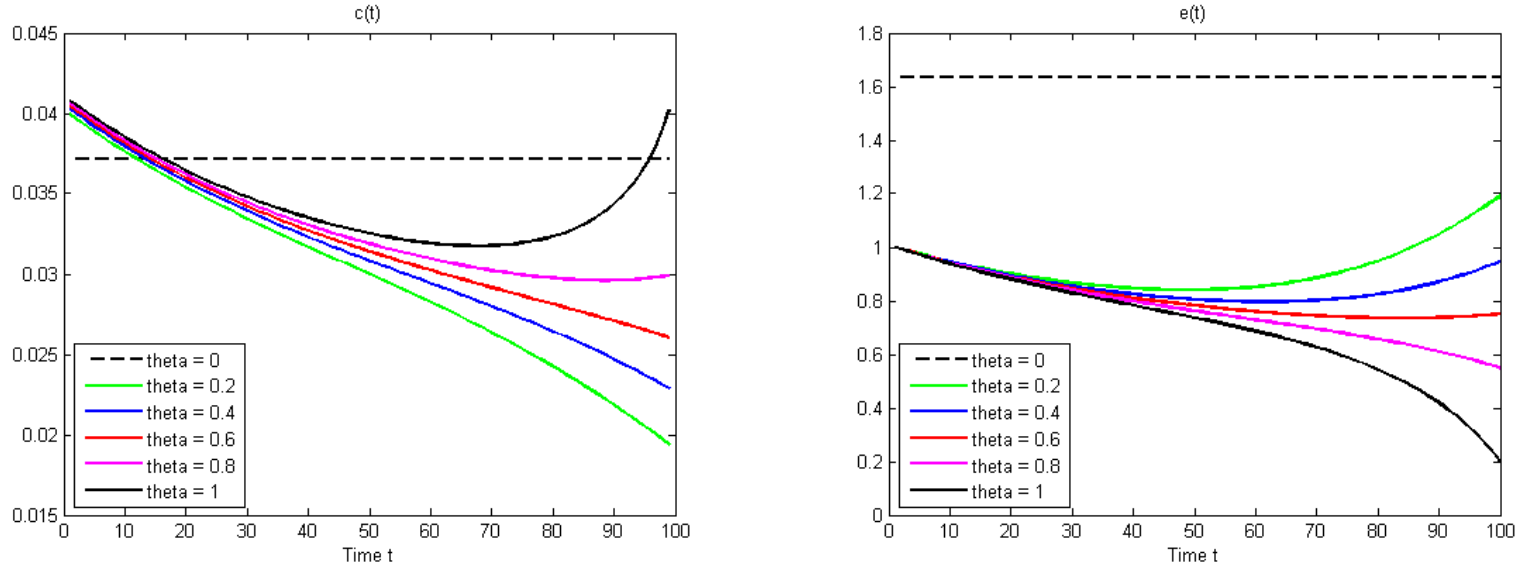

Figure 1: Scalarization technique: evolution of consumption (on the left) and natural resources (on the right) for different values of $\theta$.

Figure 2 represents the value of social welfare, $W$ (equation (6)), as a function of the parameter $\theta \in[0,1]$. Social welfare is clearly decreasing in $\theta$, meaning that it achieves its maximum when $\theta=0$, corresponding to the green golden rule case, and its minimum when $\theta=1$, corresponding to the discounted utilitarian case. Thus the green golden rule approach does yield a higher social welfare than both the Chichilnisky criterion and the discounted utilitarian approach. This result suggests that completely neglecting finite-time utilities and focusing only on the steady state utility, as suggested by the green golden rule, is not only sensible from a sustainability point of view but it is also from a social welfare maximization standpoint.

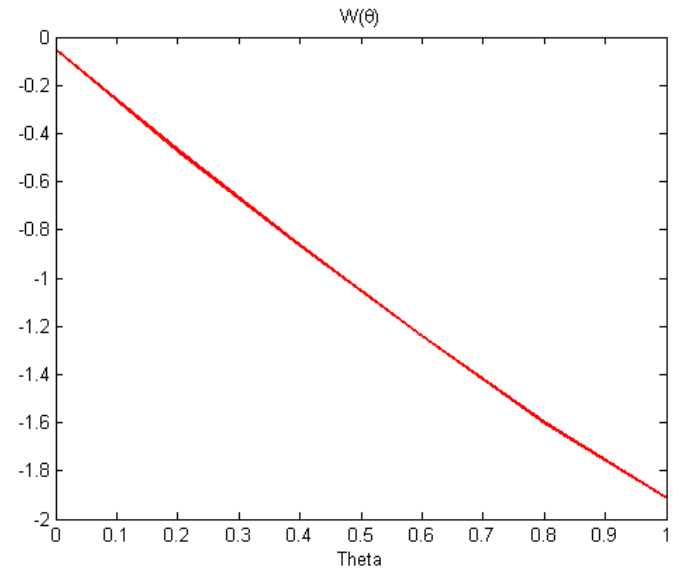

Figure 2: Scalarization technique: social welfare as a function of $\theta$.

\subsection{Goal Programming Technique}

According to the chosen GP technique, the preferences on the deviations from the fixed aspiration levels are explicitly taken into account with the help of satisfaction functions $F_{i}\left(\delta_{i}\right)$, and our multicriteria problem can be expressed as the following maximization problem:

$$
\max \quad w_{D U} F_{D U}^{+}\left(\delta_{D U}^{+}\right)+w_{D U} F_{D U}^{-}\left(\delta_{D U}^{-}\right)+w_{G G R} F_{G G R}^{+}\left(\delta_{G G R}^{+}\right)+w_{G G R} F_{G G R}^{-}\left(\delta_{G G R}^{-}\right)
$$




$$
\begin{array}{ll}
\text { s.t. } & W_{D U}-\delta_{D U}^{+}+\delta_{D U}^{-}=G_{D U} \\
& W_{G G R}-\delta_{G G R}^{+}+\delta_{G G R}^{-}=G_{G G R} \\
& 0 \leq \delta_{D U}^{+} \leq \delta_{D U \nu}^{+} \\
& 0 \leq \delta_{D U}^{-} \leq \delta_{D U \nu}^{-} \\
& 0 \leq \delta_{G G R}^{+} \leq \delta_{G G R \nu}^{+} \\
& 0 \leq \delta_{G G R}^{-} \leq \delta_{G G R \nu}^{-}
\end{array}
$$

given the dynamic equation (2), and the initial condition $e_{0}$. In the expression above $\delta_{D U \nu}^{+}, \delta_{D U \nu}^{-}, \delta_{G G R \nu}^{+}$ and $\delta_{G G R \nu}^{-}$are the veto thresholds for the positive and negative deviations from the two goals: the social planner needs to find the optimal solution satisfying these constraints on the deviations $\delta_{D U}^{+}, \delta_{D U}^{-}, \delta_{G G R}^{+}$ and $\delta_{G G R}^{-}$, respectively. The aspirations levels can be chosen freely by the social planner, in accordance to some feasibility and/or desirability criterion. In our case the most natural choice is letting the aspiration levels coincide with each goal independently considered, that is:

$$
\begin{aligned}
G_{D U}= & \max _{c_{t}} \int_{0}^{\infty} \frac{c_{t}^{1-\sigma} e_{t}^{\beta(1-\sigma)}-1}{1-\sigma} e^{-\rho t} d t \\
& \text { s.t. } \dot{e}_{t}=r e_{t}\left(1-\frac{e_{t}}{e^{c}}\right)-c_{t} \\
G_{G G R}= & \max _{c_{t}} \lim _{t \rightarrow \infty} \frac{c_{t}^{1-\sigma} e_{t}^{\beta(1-\sigma)}-1}{1-\sigma} \\
& \text { s.t. } \dot{e}_{t}=r e_{t}\left(1-\frac{e_{t}}{e^{c}}\right)-c_{t}
\end{aligned}
$$

In order to apply our GP model, we need to define the satisfaction functions $F_{i}\left(\delta_{i}^{+}\right), F_{i}\left(\delta_{i}^{-}\right)$. For sake of simplicity we assume that the shape and the properties of the satisfaction functions are the same with respect to both positive and negative deviations, and identical for each criterion. An appropriate satisfaction function may take the following form (Martel and Aouni, 1990):

$$
F_{\alpha}(\delta)=\frac{1}{1+\alpha^{2} \delta^{2}}
$$

Indeed, such a specification implies that: $F(0)=1, F(\infty)=0 ; F^{\prime \prime}(\delta)=0$ if and only if $\delta=\frac{1}{2 \alpha} ; 0.9 \leq$ $F(\delta) \leq 1$ if $\delta \leq \frac{1}{3 \alpha} ; 0 \leq F(\delta) \leq 0.1$ if $\delta \geq \frac{3}{\alpha}$. This means that this particular function allows to achieve a level of satisfaction between $90 \%$ and $100 \%$ when $\delta \leq \frac{1}{3 \alpha}$, and a level of satisfaction between $0 \%$ and $10 \%$ when $\delta \geq \frac{3}{\alpha}$. With these properties in mind, we can now proceed to define two threshold levels: the indifference threshold, $\delta_{i n d}$, and the dissatisfaction threshold, $\delta_{\text {dis }}$. A natural choice for these threshold values is the following: $\delta_{\text {ind }}=\frac{1}{3 \alpha}$ and $\delta_{\text {dis }}=\frac{3}{\alpha}$. Similarly, we need to identify the size of the veto threshold, which we assume to be given by $\delta_{v}=2 * \delta_{\text {dis }}=\frac{6}{\alpha}$. Note that the parameter $\alpha$ plays a critical role in our satisfaction function, $F_{\alpha}(\delta)$, and in particular it allows us to obtain different indifference, dissatisfaction and veto thresholds by simply changing its value. It thus may be convenient to compare our results for different choices of this parameter, for example for $\alpha=0.1, \alpha=0.01$ and $\alpha=0.001$. It is possible to show that our conclusions are not significantly affected by this parameter value, and thus in what follows we set it equal to 0.1 for the sake of simplicity (see Aouni et al., 2013).

Now we can proceed with the discretization of our problem. First of all, $G_{D U}$ and $G_{G G R}$ in a discrete framework read as follows:

$$
\begin{aligned}
G_{D U}=\max _{c_{t}} & \sum_{t=0}^{T} \frac{c_{t}^{1-\sigma} e_{t}^{\beta(1-\sigma)}-1}{1-\sigma} e^{-\rho t} \\
\text { s.t. } & e_{t+1}-e_{t}=r e_{t}\left(1-\frac{e_{t}}{e^{c}}\right)-c_{t} \quad \forall t=0, \ldots . T-1
\end{aligned}
$$




$$
\begin{aligned}
& 0=r e_{T}\left(1-\frac{e_{T}}{e^{c}}\right)-c_{T} \\
& e_{0} \text { given } \\
G_{G G R}=\max _{c_{T}} & \frac{c_{T}^{1-\sigma} e_{T}^{\beta(1-\sigma)}-1}{1-\sigma} \\
\text { s.t. } & 0=r e_{T}\left(1-\frac{e_{T}}{e^{c}}\right)-c_{T}
\end{aligned}
$$

Note that the values $G_{D U}$ and $G_{G G R}$ correspond to the cases $\theta=1$ and $\theta=0$ in equation (6), respectively. As before, some comments are needed: there is no dynamics after $T$, and the weights are set as $w_{D U}=\theta$ and $w_{G G R}=1-\theta$. The GP problem consists of maximizing the social planner's satisfaction, $Z$, and in its entire form it reads as follows:

$$
\begin{array}{ll}
\max & Z=\frac{\theta}{1+\left(\alpha \delta_{D U}^{+}\right)^{2}}+\frac{\theta}{1+\left(\alpha \delta_{D U}^{-}\right)^{2}}+\frac{1-\theta}{1+\left(\alpha \delta_{G G R}^{+}\right)^{2}}+\frac{1-\theta}{1+\left(\alpha \delta_{G G R}^{-}\right)^{2}} \\
& \sum_{t=0}^{T} \frac{c_{t}^{1-\sigma} e_{t}^{\beta(1-\sigma)}-1}{1-\sigma} e^{-\rho t}-\delta_{D U}^{+}+\delta_{D U}^{-}=G_{D U} \\
& \frac{c_{T}^{1-\sigma} e_{T}^{\beta(1-\sigma)}-1}{1-\sigma}-\delta_{G G R}^{+}+\delta_{G G R}^{-}=G_{G G R} \\
& e_{t+1}-e_{t}=r e_{t}\left(1-\frac{e_{t}}{e^{c}}\right)-c_{t} \quad \forall t=0, \ldots . T-1 \\
& 0=r e_{T}\left(1-\frac{e_{T}}{e^{c}}\right)-c_{T} \\
& e_{0} \text { given } \\
0 & \leq \delta_{D U}^{+} \leq \delta_{D U \nu}^{+} \\
0 & \leq \delta_{D U}^{-} \leq \delta_{D U \nu}^{-} \\
0 & \leq \delta_{G G R}^{+} \leq \delta_{G G R \nu}^{+} \\
0 & \leq \delta_{G G R}^{-} \leq \delta_{G G R \nu}^{-} .
\end{array}
$$
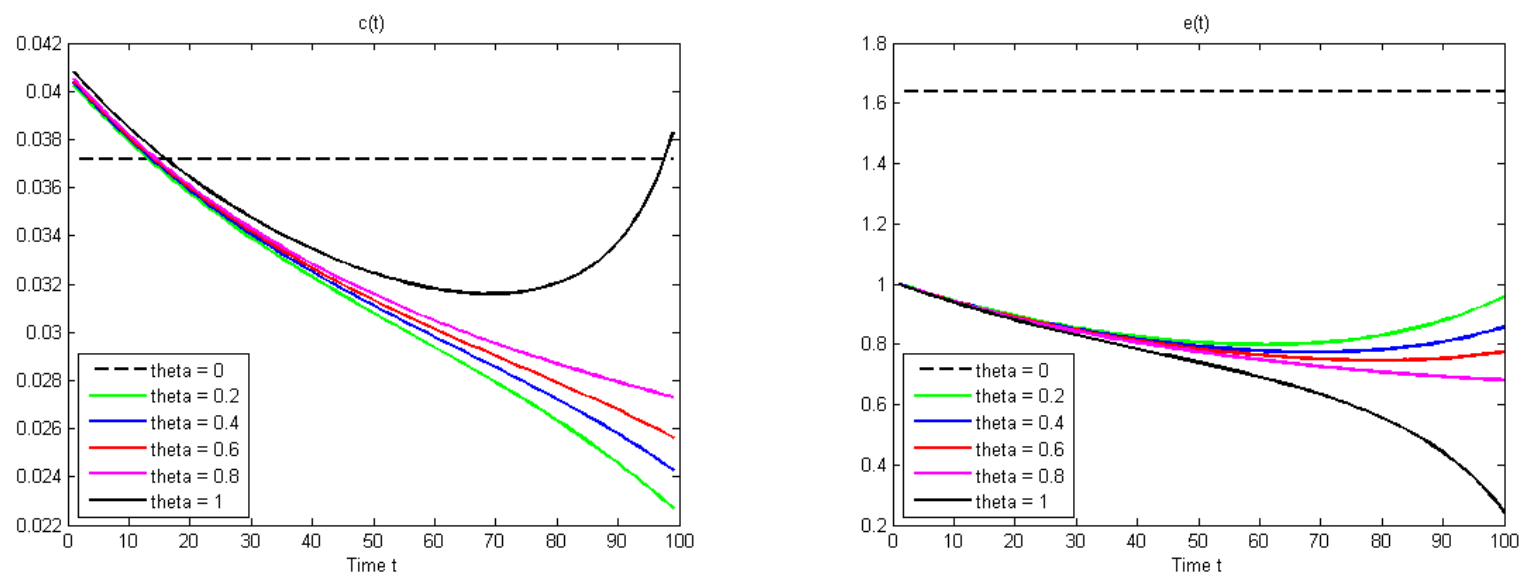

Figure 3: Goal programming: evolution of consumption (on the left) and natural resources (on the right) for different values of $\theta$.

The results of our simulations are presented in Figure 3 and in Figure 4 . The optimal time paths of consumption and natural resources are qualitatively similar to those found earlier with the scalarization approach. The dashed line still represents the green golden rule $(\theta=0)$ case, in which no dynamics from 0 to $T$ is present. On the left panel of Figure 3 we can see that consumption is generally decreasing over time 
(apart in the $\theta=1$ case), and it increases with $\theta$. The other side of the same coin can been seen on the right panel of Figure 3, the more emphasis we put on discounted utilitarianism, the less resources are left for the long run outcome, with the extreme case being represented by the discounted utilitarian approach in which no resources are left at the end of the time horizon. Since the results qualitatively coincide, their interpretation is exactly equivalent to what discussed earlier concerning the scalarization technique 4 .

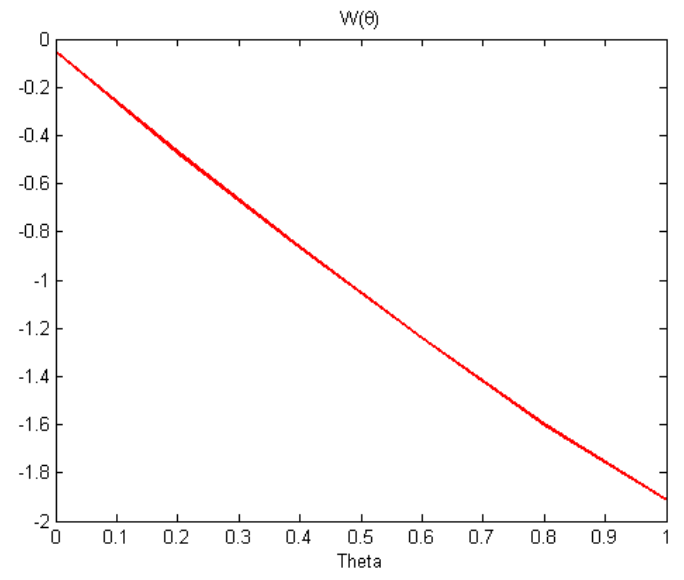

Figure 4: Goal programming: social welfare as a function of $\theta$.

Figure 4 shows the different levels of social welfare (as in equation (6), $W$, for values of $\theta$ ranging from 0 to 1 . Since the time paths of consumption and natural resources are similar to those obtained with the scalarization approach, then it is also reasonable to expect that the behavior of the social welfare function will be equivalent to what discussed earlier. This is confirmed in Figure 4 , from which we can see that social welfare is decreasing in $\theta$, and thus it achieves its maximum when $\theta=0$, corresponding to the green golden rule criterion. Even with a GP approach, our results confirm that the green golden rule is not only the preferred criterion from a sustainability point of view but also from a welfare maximization standpoint.

\section{Conclusion}

In (macro)economics literature the notion employed in order to define social welfare is critical, especially whenever we wish to take into account also sustainability issues. This is due to the fact that the most commonly used criterion, namely the discounted utlitarianism, attaches less weight to future generations' wellbeing. Alternative criteria, like the green golden rule and the Chinchilnisky criterion, have been proposed in order to overcome this problem. In this paper we assess and compare the outcomes associated to different welfare criteria by simply relying on a multicriteria model. We consider two alternative specification of the multicriteria problem, based on the scalarization and GP technique respectively, in order to fully analyze the problem in a very simple macroeconomic model with environmental interactions, as in Chichilnisky et al. (1995). For a specific and realistic parametrization of the model, we show that social welfare is a decreasing function of the weight attached to the discounted sum of utilities $(\theta)$. Thus, it is possible to identify a clear ranking of criteria in terms of social welfare achievements: the green golden rule leads to the highest level of welfare, the Chichilnisky criterion to an intermediate level, and the discounted utilitarianism to the lowest welfare level. Both the scalarization and the GP techniques, even if relying on different multicriteria

\footnotetext{
${ }^{4}$ The scalarization and the GP approach coincide when the objectives values of the GP are chosen exactly equal to each objective taken singularly. When we introduce the preferences via the satisfaction function the outcome of the two approaches is generally different and the similarities occurring in our results are given by the above mentioned matching of the GP objectives with each objective taken singularly.
} 
philosophies, lead to the same results (see Figure 2 and Figure 44. Such concordant conclusions corroborate a result that is absolutely not obvious a priori: the green golden rule does have to be preferred to the discounted utilitarianism not only because of its ability to take into account sustainability issues, but also because it allows to achieve higher social welfare levels.

To the best of our knowledge, ours is the first paper trying to assess the implications of different notions of social welfare on the economic and environmental trade off. It is thus natural to wonder to what extent the simplicity of our model specification affects our results. Specifically, in our model natural resources are only used for consumption purposes; however, this is quite a strong simplification of reality. Natural resources represent also an important production factor, without which it may not be possible at all to produce any consumable good. Moreover, production activities, apart from using natural resources, may even be detrimental for the environment through the pollution channel. Furthermore, pollution may generate perverse effects on the production possibilities, thus the nexus between economic activities and environmental dynamics is definitely more complex that what we have considered thus far. Extending the analysis along these directions is needed in order to obtain a wider comprehension of sustainability issues and their implications on social welfare. This is left for future research.

\section{References}

1. André, F.J., Cardenete, M.A., Romero, C. (2009). A goal programming approach for a joint design of macroeconomic and environmental policies: a methodological proposal and an application to the Spanish economy, Environmental Management 43, 888-898

2. Aouni, B., Kettani, O. (2001). Goal programming model: a glorious history and a promising future, European Journal of Operational Research 133, 225-231

3. Aouni, B., Colapinto, C., La Torre, D. (2013). A cardinality constrained stochastic goal programming model with satisfaction functions for venture capital investment decision making, Annals of Operations Research 205, 77-88

4. Aouni, B., La Torre, D. (2010). A generalized stochastic goal programming model, Applied mathematics and computation 215, 4347-4357

5. Aouni A., Ben Abdelaziz, F., La Torre, D. (2012a). The stochastic goal programming model: theory and applications, Journal of Multicriteria Decision Analysis 19, 185-200

6. Aouni, B., Colapinto, C., La Torre, D. (2012b). Stochastic goal programming model and satisfaction function for media selection and planning problem, International Journal of Multi-criteria Decision Making 2, 391-407

7. Arrow, K., Dasgupta, P., Goulder, L., Daily, G., Ehrlich, P., Heal, G., Levin, S., Maler, K.G., Schneider, S., Starrett D., Walker, B. (2004). Are we consuming too much?, Journal of Economic Perspectives 18, $147-172$

8. Barro, R.J., Sala-i-Martin, X. (2004). Economic growth (Cambridge, Massachusetts: MIT Press)

9. Boucekkine, R., Fabbri, G. (2013). Assessing Parfit's repugnant conclusion within a canonical endogenous growth set-up, Journal of Population Economics 26, 751-767

10. Charnes, A., Cooper, W.W. (1959). Chance-constrained programming, Management Science 6, 73-80

11. Charnes, A., Cooper, W.W. (1968). Deterministic equivalents for optimising and satisfying under chance constraints, Operations Research 11, 11-39

12. Charnes, A., Cooper, W.W., Ferguson, R. (1955). Optimal estimation of executive compensation by linear programming, Management Science 1, 138-151

13. Chinchilnisky, G., Heal, G., Beltratti, A. (1995). The green golden rule, Economics Letters 49, 174-179

14. Chinchilnisky, G. (1997). What is sustainable development?, Land Economics 73, 476-491 
15. Eliasson, L. Turnovsky, S.J. (2004). Renewable resources in an endogenously growing economy: balanced growth and transitional dynamics, Journal of Environmental Economics and Management 48, 1018-1049

16. Figuieres, C., Tidball, M. (2012). Sustainable exploitation of a natural resource: a satisfying use of Chichilnisky's criterion, Economic Theory 49, 243-265

17. Gamper, C.D., Turcanu, C. (2007). On the governmental use of multi-criteria analysis, Ecological Economics 62, 298-307

18. Guitouni, A., Martel, J.M. (1998). Tentative guidelines to help choosing an appropriate MCDA method, European Journal of Operational Research 109, 501-521

19. Heal, G. (2005). Intertemporal welfare economics and the environment, in Maler, K.G., Vincent, J.R. (eds.), "Handbook of Environmental Economics", vol. 3 (North-Holland: Amsterdam)

20. Hwang, C.L., Masud, A.S. (1979). Multiple objective decision making, methods and applications: a state-of-the-art survey (Springer-Verlag)

21. Janssen, R. (2001). On the use of multi-criteria analysis in environmental impact assessment in the Netherlands, Journal of Multi-Criteria Decision Analysis 10, 101-109

22. Le Kama, A.D.A. (2001). Sustainable growth, renewable resources and pollution, Journal of Economic Dynamics \& Control 25, 1911-1918

23. Marsiglio, S. (2011). On the relationship between population change and sustainable development, Research in Economics 65, 353-364

24. Marsiglio, S. (2014). Reassessing Edgeworth's conjecture when population dynamics is stochastic, Journal of Macroeconomics 42, 130-140

25. Marsiglio, S., La Torre, D. (2012). Population dynamics and utilitarian criteria in the Lucas-Uzawa model, Economic Modelling 29, 1197-1204

26. Martel, J.M., Aouni, B. (1990). Incorporating the decision-maker's preferences in the goal-programming model, Journal of the Operational Research Society 41, 1121-1132

27. Mohtadi, H. (1996). Environment, growth, and optimal policy design, Journal of Public Economics $63,119-140$

28. Nijkamp, P., van Delft, A. (1977). Multicriteria analysis and regional decisionmaking (Boston: Kluwer Nijhoff)

29. Palivos, T., Yip, C.K. (1993). Optimal population size and endogenous growth, Economics Letters 41, $107-110$

30. Pezzey, J.C.V. (1997). Sustainability constraints versus "optimality" versus intertemporal concern, and axioms versus data, Land Economics 73, 448-466

31. Ramsey, F. (1928). A mathematical theory of saving, Economic Journal 38, 543-559

32. Romero, C. (1991). Handbook of critical issues in goal programming (Pergamon Press, Oxford)

33. Sawaragi, Y., Nakayama, H., Tanino, T. (1985). Theory of multiobjective optimization, (Orlando: Academic Press)

34. Shmelev, S.E. (2011). Dynamic sustainability assessment: the case of Russia in the period of transition (1985-2007), Ecological Economics 70, 2039-2049

35. Steuer, R.E. (1986). Multiple criteria optimization: theory, computation, and application (New York: Wiley \& Sons)

36. World Commission on Environment and Development (1987). Our common future (Oxford University Press, Oxford) 